\title{
基于高斯映射的可展曲面等曲率离散问题研究
}

\author{
张利萍 1,2 彭彦平 2 \\ (1. 西湖大学工学院 杭州 310024; \\ 2. 大连工业大学机械工程与自动化学院 大连 116034)
}

\begin{abstract}
摘要: 提出可展曲面及其折展结构的空间高斯映射数学建模方法与分析理论, 实现空间折展特性分析与折纸曲面的离散化研 究, 并继而可以进一步引申到可展曲面的等效数学模型的空间机构学研究。通过建立可展曲面的高斯映射方法, 建立空间折 展曲面与复杂折展结构的高斯球面曲线, 实现三维空间折展结构降维到二维球面曲线, 且以高斯映射方法建立可展折纸曲面 的数学模型基础理论, 并通过曲线曲面理论, 将三维的可展曲面问题转化为高斯球面曲线的数学分析。继而通过折展曲面与 折展单元的高斯球面曲线的对应研究分析, 且由球面曲线的离散得到折纸曲面的近似多面体折展结构, 并为其等效机构学特 性研究奠定基础, 进一步将曲面折展转化为平面折展可对应的现有机构和机器人学问题, 实现曲面折展问题与平面折展问题 对接, 并探讨其工程应用依据。
\end{abstract}

关键词: 曲面折展; 可展曲面; 高斯映射; 曲面离散; 曲率 中图分类号: TG156

\section{Gauss Map Based Developable Surface Discretization}

\author{
ZHANG Liping ${ }^{1,2}$ PENG Yanping ${ }^{2}$
}

(1. School of Engineering, Westlake University, Hangzhou 310024;

2. Department of Mechanical Engineering, Dalian Polytechnic University, Dalian 116034)

\begin{abstract}
Gauss map of the continuous curved surfaces is lent to model the geometry properties and configuration information of spatial curved origami. The Gauss spherical curves achieve the goal of dimension deduction of the discretization problem in which $R^{3}$ surface discretization is shifted to spherical curve discretization. Then, the equal segments are utilized to discretize the spherical curves first. The resulting points on the spherical curves present the discretized normal directions of the curved surface. Thus, the discretized ruling lines, as new folds in the approximate polyhedron, can be obtained. The aim of this work has been to provide a curvature discretization for developable surfaces based on the Gauss spherical curves. The directed foldable units are identified and the discrete operations of these units are proposed. More complex curved origami can be analyzed with its decomposition and the constructive units. It provides a differential geometry means to deal with the instantaneous folding movements. Folding with non-flat curvature is exploited. The equal curvature discretization of curved surface is realized.
\end{abstract}

Key words: curved origami; developable surface; Gauss map; curved discretization; curvature

\section{0 前言}

传统折纸大多是沿着直线折叠 ${ }^{[1-4]}$, 但通过将直 线折痕转化为曲线折痕, 可使平面单元和多面体面 转化为曲面单元和可展曲面, 这些可展曲面是具备 灵活运动的折纸表面, 可以模拟空间物体的折展运 动, 如类似金属板、纸张、胶合板、皮革、织物等 材料的折展。1976 年, HUFFMAN ${ }^{[5]}$ 给出了关于零曲 率曲面在雉面的折痕和顶点附近是如何运动的数学

20190408 收到初稿, 20191016 收到修改稿
描述, 这些实体是分段平面的边和顶点的概括, 并 研究了任意多面体顶点平面扇形区域在高斯球面上 的表示, 这对于可展曲面的设计及其数学理论研究 有着指导意义, 无论是作为艺术品还是作为数学研 究。FUCHS 等 ${ }^{[6]}$ 对可展曲面和弯曲折痕的理解, 尤 其是曲面折痕与曲面之间的数学关系的建立做出了 进一步的重要贡献。在文献[7]中, 用圆雉单元建立 了可展曲面的一个简单近似。 $\mathrm{BO}$ 等 ${ }^{[8]}$ 提出了一种新 颖有效的可展曲面建模方法用来模拟纸张弯曲的相 互作用和三维应用。通过操纵测地线, 为纸张弯曲 的建模提供直观的形状控制。KOSCHITZ ${ }^{[9]}$ 讨论了与 折纸中曲线折叠有关的一些基本结果, 部分理论可 
以从微分几何的概念和理论中导出。KILIAN 等 ${ }^{[10]}$ 利用平面四边形网格(PQ-meshes)对曲面折叠进行建 模, 并部署了一种基于优化的方法。KERGOSIEN 等 ${ }^{[11]}$ 采用工程方法对折纸进行了早期的模拟。从一 般曲线出发, 它们能够拟合可展曲面。在文献[12]中, 基于理想的三维柱面提出了一种二维 Miura-ori 嵌入 的平面设计方法。 $\mathrm{TACHI}{ }^{[13]}$ 通过组装弯曲折叠的薄 板, 设计了一个可展开的单元管状结构。通过对刚 性折纸结构的细化, 得到了曲面的单自由度机构。

DUDTE 等 ${ }^{[14]}$ 的研究展示出无需测量的基本几何结 构和约束优化算法, 这可以用来确定空间变换的算 法, 这些算法可给出近似的曲率变化曲面。文献[15] 中, 提出了一种利用层状阵列网络对大曲率和可展 结构进行数学建模和设计的方法。

在本文中提出了一种等曲率离散的算法, 该算 法在给定曲面折展的情况下, 产生一个折展曲面的 近似多面体这展结构, 包含曲面折展的离散化是将 每个弯折的曲面用一组直线折痕与小平面片所取 代。由于可展曲面的特殊曲面性质, 即与沿着直母 线方向的法矢方向相同, 因此利用高斯映射进行曲 面的降维映射, 将三维的折展问题转化为二维的高 斯球面曲线问题。可展曲面的离散, 不是简单地近 似光滑表面的几何性质, 而是利用高斯球面曲线的 性质对可展曲面的曲率性质的操作。该方法应用在 折纸特别是在曲面折纸研究中, 使得直线折展得到 更广泛的拓展, 而可展曲面的等曲率离散化可以将 直线折展的潜在知识继续深入到曲面折展机构的研 究中。

\section{1 可展曲面与高斯映射}

\section{1 可展曲面}

可展曲面是在其上每一点处高斯曲率为零的曲 面。具有常数高斯曲率的曲面能够经弯曲(非拉伸、收 缩、刍褶或撕裂)而变为任何一个具有相同常数高斯曲 率的曲面。因为平面就是在每一点处高斯曲率为常数 零的特殊曲面, 所以每一点处曲率为零的任一曲面, 能够经弯曲而展开成平面。在此讨论的常用可展曲面 包含平面、圆柱面、圆雉面以及切线面, 这些曲面由 通过准线的直母线运动形成, 如图 1 所示。

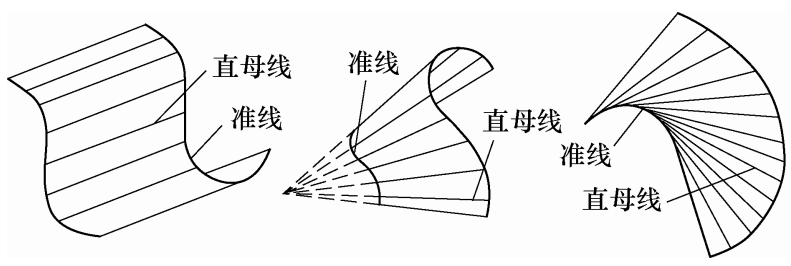

图 1 三种常用可展曲面

\section{2 曲面折展单元}

基本可展曲面的组合连接是可展曲面单元形成 的基础。两个基本可展曲面的连接方式主要有两种: 其一, 两个基本可展曲面由折痕曲线相交连接, 所 以最简单的折展单元是由左右两个折展曲面与连接 其的折痕曲线组成，在此称其为串接式。其二，两 个基本可展曲面由共用直母线的方式相连接而形成 的组合单元, 定义为并接式。本文主要以不含扭转 曲线的折展单元为研究对象, 如图 2 所示。
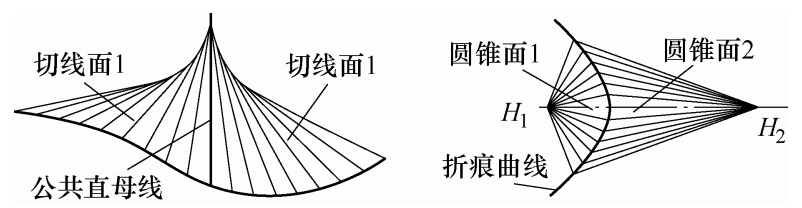

图 2 两类可展基本单元

\section{3 高斯映射}

将物体表面每点的法向矢量映射到一个单位球 上，这个球称为高斯参考球。高斯映射是将曲面 $\Sigma$ 上点 $P$, 其单位法矢量 $\boldsymbol{n}(|\boldsymbol{n}|=1)$, 将矢量 $\boldsymbol{n}$ 的起点平 行地移到原点 $O$ 后, $\boldsymbol{n}$ 的终点就是以在 $O$ 点为球心 的单位球面 $S^{2}$ 上一点, 于是把这种点的法向矢量映 射称为其法向在高斯球上的高斯映射, 如图 3 所示。
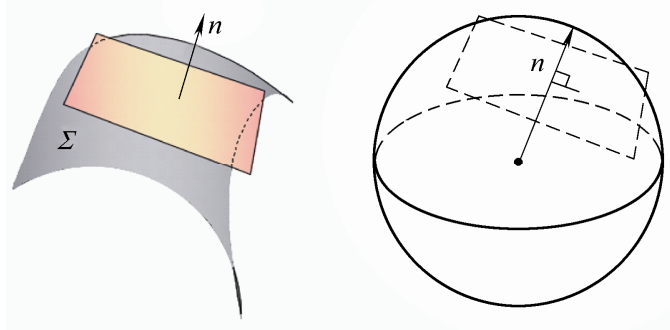

图 3 高斯映射

基于高斯映射的概念，可以进一步建立可展曲 面的高斯球面曲线，实现三维的空间曲面转换为二 维球面曲线的几何模型。

\section{2 高斯球面曲线}

由于可展曲面是由直母线的连续运动产生, 且 沿着每条直母线, 仅有唯一切线面, 这意味着沿任 一直母线, 其上点的法矢方向不变, 即仅有同一单 位法矢。

\section{1 可展曲面及其高斯球面曲线}

依次建立可展曲面的高斯球面曲线如图 4 所 示, 图 $4 \mathrm{a} \sim 4 \mathrm{c}$ 分别为圆柱面、圆雉面和切线面的高 斯映射过程。

圆柱面在高斯球上的高斯映射形成一个大圆球 面曲线, 圆雉面的映射为一个小圆球面曲线, 切线 面的则为一般性的球面曲线。 


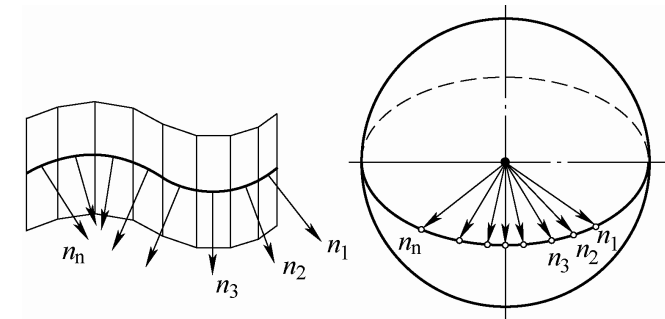

(a)
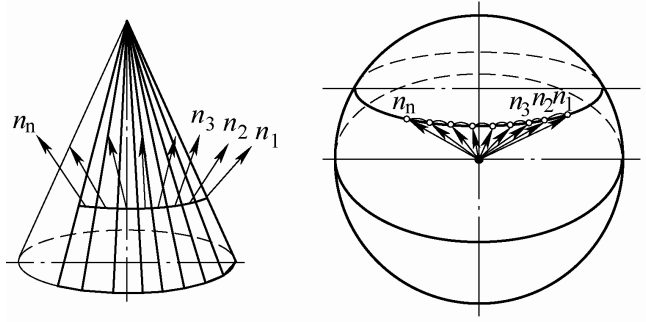

(b)
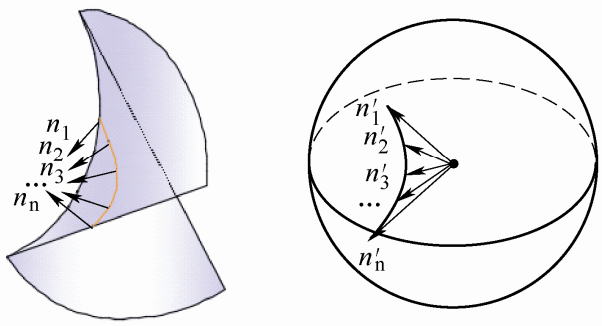

(c)

图 4 可展曲面的高斯映射

\section{2 曲面折展单元及其高斯球面曲线}

复杂曲面折展结构可由构成其折展单元决定, 因此分析最简曲面折展单元的高斯映射对于折展结 构的整体性质研究尤为重要。

对于串接式曲面折展单元, 其折痕曲线为 $P_{1}$ $P_{2}$, 连接的两个可展曲面分别为圆柱面 $\Sigma_{1}$ 和圆雉 面 $\Sigma_{2}$, 如图 5 所示。每个曲面有各自的高斯映射 球面曲线。 $\Sigma_{1}$ 对应的是大圆球面曲线 $C_{1}$, 有映射 $\mathrm{N}_{1}$ : $\Sigma_{1} \rightarrow C_{1} ; \Sigma_{2}$ 对应的是小圆球面曲线 $C_{2}$, 对应映射 $\mathrm{N}_{2}: \Sigma_{2} \rightarrow C_{2}$ 。
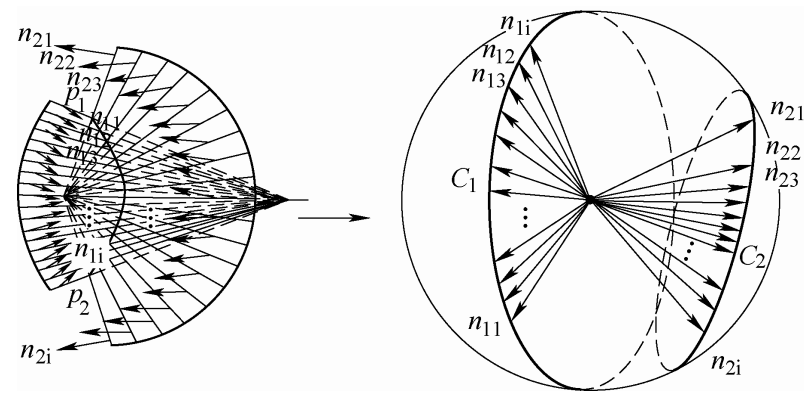

图 5 串接式曲面折展单元及其高斯映射

考虑到并接式的曲面相接组合的特点, 以圆柱 与圆雉的曲面单元组合为例, 如图 6 所示可以得到 其曲面折展单元的高斯球面曲线。
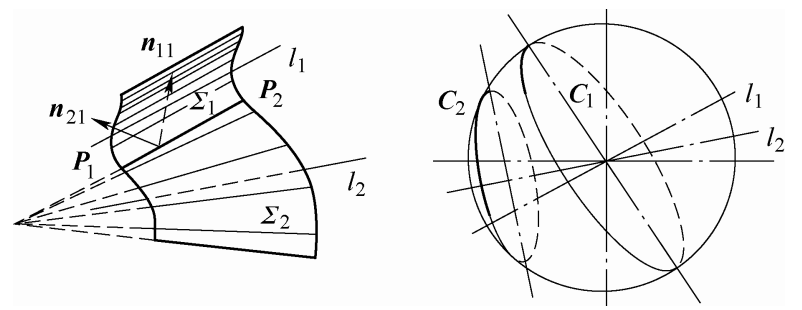

图 6 并接式曲面折展单元及其高斯映射

\section{3 高斯球面曲线特性分析}

由于通过高斯映射已经将 $\boldsymbol{R}^{3}$ 空间的三维折展 结构降维到 $\boldsymbol{S}^{2}$ 空间的球面曲线, 因此折展结构的分 析和进一步研究可以通过球面曲线的几何空间性质 和映射特征等结合起来研究。分析高斯球面曲线的 特性, 有如下性质。

(1) 球面高斯曲线是可展曲面上直母线的连续 法矢的映射, 其映射与通过可展曲面的曲线无关。

(2) 以图 7 的两个圆雉面及相连接的折痕曲线 $P Q$ 为例, 高斯球面曲线 $C_{1}$ 与 $C_{2}$ 的几何关系及其折 展特性可归纳如下。

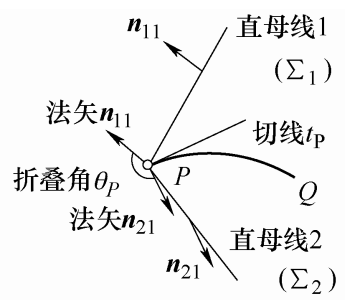

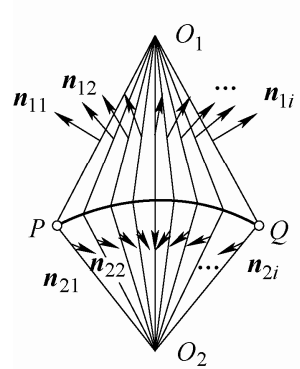

(a)

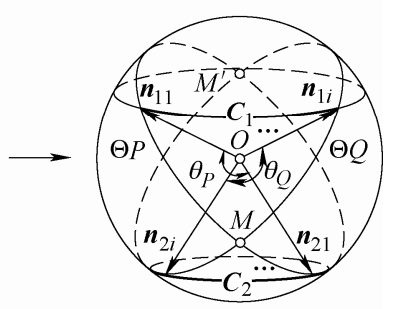

(b)
图 7 折叠角与高斯球面曲线

(1) 在图 7a 中, 折痕曲线上的点 $P$ 处有两个单 位法矢 $\boldsymbol{n}_{11}$ 和 $\boldsymbol{n}_{21}$, 分别为曲面 $\Sigma_{1}$ 和 $\Sigma_{2}$ 在 $P$ 点的法 矢, 同时有 $P$ 点的切矢 $\boldsymbol{t}_{P}$ 垂直于两个相交法矢 $\boldsymbol{n}_{11}$ 和 $\boldsymbol{n}_{21}$ 。由于法矢 $\boldsymbol{n}_{11}$ 和 $\boldsymbol{n}_{21}$ 确定一个法平面, 则 $\boldsymbol{t}_{P}$ 垂直于这个平面。如图 8 所示。同样在 $Q$ 点, 可得 到 $\boldsymbol{t}_{Q}$ 垂直于其法矢的平面, 由此沿折痕曲线的连续 变化点, 有球面曲线 $\overparen{t_{P} t_{Q}}$, 该曲线对应沿折痕曲线 切矢连续变化。

(2) 在图 7b 中, 法矢 $\boldsymbol{n}_{11}$ 和 $\boldsymbol{n}_{21}$ 作为单位高斯球 的半径, 能够确定一个大圆平面 $\odot P$, 在这个平面 


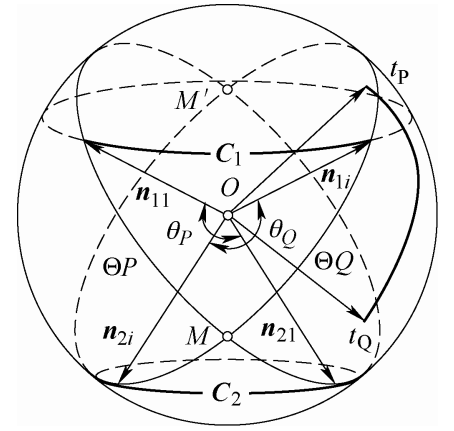

图 8 切矢与法矢所在的大圆面及其对应关系

上, 法矢 $\boldsymbol{n}_{11}$ 和 $\boldsymbol{n}_{21}$ 中间所夹的角度即为 $P$ 点处的折 叠角, 记为 $\theta_{P}$ 。在单位高斯球中, 大圆弧 $\overline{\boldsymbol{n}_{11} \boldsymbol{n}_{21}}$ 可 表示折叠角 $\theta_{P}$ 大小。

(3) 对于折痕曲线上的每一个点, 都有相连接的 两个曲面上的直母线与之对应, 即都可形成系列的 大圆, 随折痕曲线上的点的连续变化, 这些大圆也 对应形成连续变化。

(4) 如果折痕曲线的挠率为零, 则意味着该曲线 的密切面唯一, 即球面曲线 $\widetilde{\boldsymbol{t}_{P} \boldsymbol{t}_{Q}}$ 为大圆弧曲线。点 $M$ (或 $M^{\prime}$ ) 为该密切面的法矢, 且平分球面曲线 $\widetilde{\boldsymbol{n}_{11} \boldsymbol{n}_{21}}$ 与 $\overline{\boldsymbol{n}_{1 i} \boldsymbol{n}_{2 i}}$ 。对应三维折展结构性质则是该密切面的法 矢平分折叠角的大小。

(3) 高斯映射是瞬态映射, 对应一个折叠的瞬 时状态, 关于映射的计算都可以通过曲面的方程得 来。一个瞬时的折叠状态对应一个具体方程形式, 这个性质可以扩展到基于瞬态的可展曲面的离散 研究。

\section{4 可展曲面结构的离散}

基于前面的高斯映射性质分析, 可知三维折展 结构的相关折叠信息都可以转化到高斯球面曲线来 操作, 于是可以利用高斯曲线性质来研究可展结构 的离散化问题。

\section{1 可展曲面的离散问题}

对于可展曲面的离散化问题, 可转化为其直母 线的离散。由于已知直母线的法矢映射为高斯球面 曲线, 则基于高斯曲线的离散化过程如图 9 所示, 步骤如下。

(1) 建立可展曲面的连续法矢。以切线面如图 9 为例, 由切线面的连续直母线, 可建立其相对应 的切线面 $\left\{T_{1}, \cdots, T_{i}, T_{j}\right\}$, 继而由切线面建立起法矢 集合。

(2) 高斯映射。将切线面的法矢映射到高斯 球内, 单位法矢的末端形成高斯球面曲线, 由此 切线面的性质分析可转移到其高斯球面曲线性 质分析。
(3) 球面曲线的离散。曲面的曲率特征已经映 射到高斯球面曲线, 由此可以离散球面曲线为可展 曲面的离散对象。又因需要处理的是曲面的等曲率 变化离散, 则在离散球面曲线时, 采用等圆心角 $\Delta \theta$ 来得到连续的离散点。在单位球内, 密集的离散变 量 $\Delta \theta$ 可以由等弧长 $\Delta l$ 近似替代。

(4) 将离散的法矢方向映射回可展曲面, 离散 后的球面曲线上的离散点代表了可展曲面上的被离 散法矢的方向。

(5) 得到离散的直母线。被离散的法矢方向直 接对应着曲面的直母线的方向，继而连接离散的 直母线以及折痕曲线的离散点, 可得近似的多面 体结构。

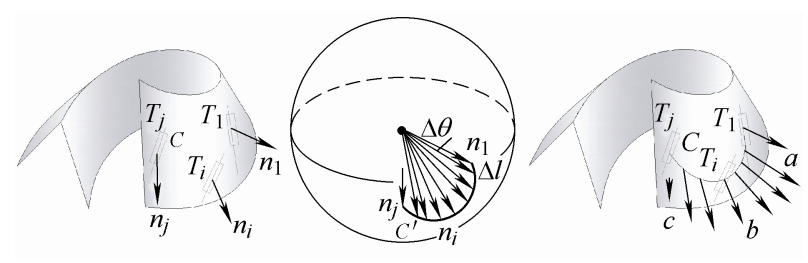

图 9 切线面离散过程分解

该过程将可展曲面的等曲率离散转为高斯曲线 的离散问题，并通过反映射得到其近似多面体结构。 由于对应的是等曲率的离散, 这种方法实现了由可 展曲面均匀地向平面的近似, 在曲率变化较快的部 分, 近似平面增多, 反之, 曲率变化较慢的部分, 近似平面数量较少。

\section{2 可展单元的离散问题}

可展单元由单个可展曲面组成, 其离散问题可 以归由可展曲面的离散。由于在折痕曲线上, 每个 点连接左右两个曲面, 因此可以选择任一曲面做离 散点的操作起始曲面, 则另一个曲面的离散直母线 会由起始曲面经过折痕曲线相应地确定下来。

折痕曲线 $P_{1} P_{2}$ 连接两个圆雉曲面 $\Sigma_{1}$ 与 $\Sigma_{2}$, 如 图 10a 所示。其中 $\Sigma_{1}$ 对应大圆球面曲线 $C_{1}$, 有映射 $\mathrm{N}_{1}: \Sigma_{1} \rightarrow C_{1} ; \Sigma_{2}$ 对应小圆球面曲线 $C_{2}$, 对应映射 $\mathrm{N}_{2}: \Sigma_{2} \rightarrow C_{2}$, 如图 10b 所示。通过离散 $\Sigma_{1}$ 或 $\Sigma_{2}$, 可得到整个单元相应的直母线离散, 实现构成曲面 的离散, 如图 10c、10d 所示。

在单元的离散过程中, 折痕曲线与其两侧连接 的曲面离散有着一一对应关系。原因如图 $10 \mathrm{~b}$ 所示, $P$ 点处法矢 $\boldsymbol{n}_{11}$ 和 $\boldsymbol{n}_{21}$ 作为单位高斯球的半径, 在确 定的大圆平面上，法矢 $\boldsymbol{n}_{11}$ 和 $\boldsymbol{n}_{21}$ 对应，这种性质同 样地可以顺延到整个的折痕曲线, 因此折痕曲线两 侧的法矢离散也有着对应关系，基于此才可以得到 两个连接曲面与折痕曲线这三个元素的离散, 可以 获得另外两个元素的离散，其间具有相通性。 


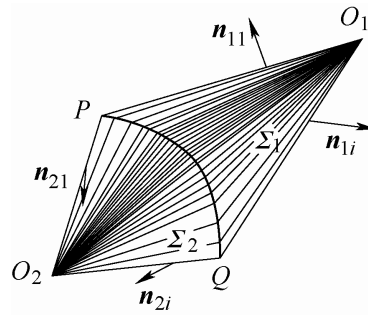

(a)

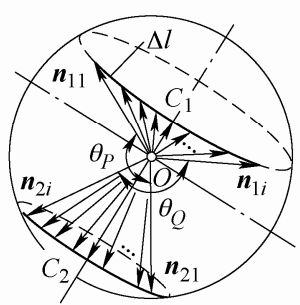

(c)

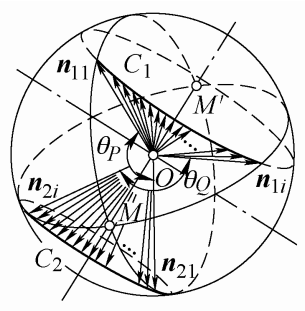

(b)

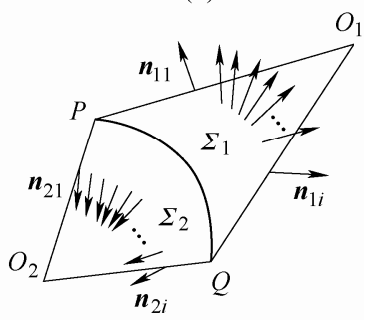

(d)
图 10 可展单元离散过程示意

\section{3 折痕曲线的离散与曲面离散的转化关系}

可展曲面与可展单元的离散均是通过离散可展 曲面的法矢来实现基于等曲率变化的离散。在上一 节中可知两个连接曲面与折痕曲线这三个元素中已 知其中一个元素的离散, 另外两个具有对应性, 可 分别获得。

以曲线折痕为例, 其等曲率的离散等价于其切 线等变化率的离散。折痕曲线的离散与可展曲面的 离散的关系是折痕的离散可以看成上述连续大圆平 面间的离散, 等同于切矢曲线 $\widetilde{t_{P} t_{Q}}$ 的离散, 其结果 相同。

\section{5 折叠角与可展多面体近似}

\section{1 折叠角}

对于可展曲面上的曲线 $\Gamma$ 有

$$
\Gamma: \boldsymbol{r}=\boldsymbol{c}(u) \quad u_{0} \leqslant u \leqslant u_{1}
$$

假设该曲线与所有直母线均相交, 非零矢量 $\boldsymbol{l}(u)$ 通过点 $\boldsymbol{c}(u)$, 可得可展曲面的方程 $\Sigma$ 为

$$
\begin{gathered}
\Sigma: \boldsymbol{D}(u, v)=\boldsymbol{c}(u)+v \cdot \boldsymbol{l}(u) \\
u_{0} \leqslant u \leqslant u_{1},-\infty \leqslant v \leqslant+\infty
\end{gathered}
$$

若可展曲面 $\Sigma_{1}$ 与 $\Sigma_{2}$ 的 Frenet 标架如图 14 所示, 可表示为

$$
\Sigma_{1}:\left\{Q ; e_{1}, e_{2}, e_{3}\right\} ; \Sigma_{2}:\left\{Q ; e_{1}^{\prime}, e_{2}^{\prime}, e_{3}^{\prime}\right\}
$$

则其折叠角 $\beta$ 为

$$
\begin{gathered}
\beta=\arccos \left(\frac{e_{3} \cdot e_{3}^{\prime}}{\left|e_{3}\right| \cdot\left|e_{3}^{\prime}\right|}\right) \\
\boldsymbol{e}_{1}=\boldsymbol{l}, \boldsymbol{e}_{2}=\frac{\mathrm{d} \boldsymbol{l} / \mathrm{d} u}{|\mathrm{~d} \boldsymbol{d} / \mathrm{d} u|} \quad \boldsymbol{e}_{3}=\boldsymbol{e}_{1} \times \boldsymbol{e}_{2}=\boldsymbol{l} \times \frac{\mathrm{d} \boldsymbol{l} / \mathrm{d} u}{|\mathrm{~d} \boldsymbol{d} u|}
\end{gathered}
$$

$$
\boldsymbol{e}_{1}^{\prime}=\boldsymbol{l}^{\prime}, \boldsymbol{e}_{2}^{\prime}=\frac{\mathrm{d} \boldsymbol{l}^{\prime} / \mathrm{d} u}{\left|\mathrm{~d} \boldsymbol{l}^{\prime} / \mathrm{d} u\right|} \quad \boldsymbol{e}_{3}^{\prime}=\boldsymbol{e}_{1}^{\prime} \times \boldsymbol{e}_{2}^{\prime}=\boldsymbol{l}^{\prime} \times \frac{\mathrm{d} \boldsymbol{l}^{\prime} / \mathrm{d} u}{\left|\mathrm{~d} \boldsymbol{l}^{\prime} / \mathrm{d} u\right|}
$$

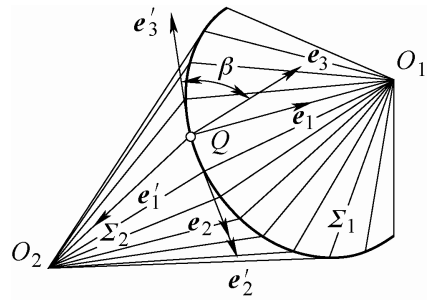

图 11 折叠角与折展单元

\section{2 可展曲面的多面体近似}

可展曲面中的圆柱和圆雉曲面离散以后可形成 棱柱和棱雉, 均为可展的多面体结构。对于切线面 的离散近似多面体结构则比较复杂。对于任意切线 面如图 12a 所示, 可以得到其离散的直母线形式如 图 $12 \mathrm{~b}$ 所示, 由于切线面的直母线的离散为异面直 线的形式，所以其最小离散后的多面体结构为异面 四边形结构, 如图 12c 所示。其中相对应的边 $P^{\prime} Q^{\prime}$ 与 $P Q, P P^{\prime}$ 与 $Q Q^{\prime}$, 及异面对角线 $P^{\prime} Q^{\prime}$ 与 $P Q$ 构成 了四面体 $P P^{\prime} Q Q^{\prime}$ 。同前面分析, 在折痕 $M$ 点处, 其折叠角 $\beta_{M}$ 可由两侧的法矢 $\boldsymbol{n}_{L}$ 和 $\boldsymbol{n}_{R}$ 计算得出。异 面四边形可以展开成图 $12 \mathrm{~d}$ 。

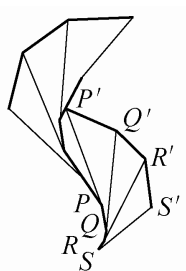

(a)

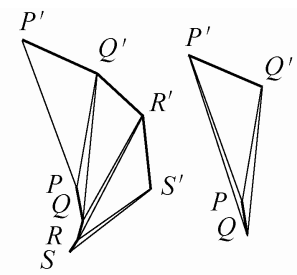

(b)

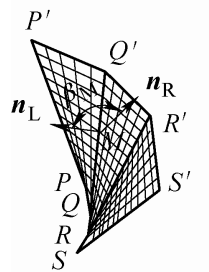

(c)

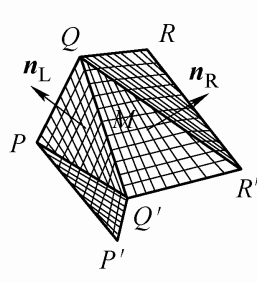

(d)
图 12 切线面及其离散多面体结构

可见不同可展曲面具有不同多面体近似特性。

6 算例: 切线面的高斯映射及其离散

首先圆柱面与圆锥面相交，以产生切线面的准 线方程, 如图 13 所示。

其中圆柱面的方程为

$$
\left\{\begin{array}{l}
x=\cos ^{2} \theta \\
y=\cos \theta \sin \theta \quad 0 \leqslant \theta \leqslant 2 \pi \\
-2 \leqslant z \leqslant 2
\end{array}\right.
$$




\section{圆雉面的方程为}

$$
\left\{\begin{array}{l}
x=z \sin ^{2} \theta \\
y=z \cos \theta \quad 0 \leqslant \theta \leqslant 2 \pi \\
-1 \leqslant z \leqslant 1
\end{array}\right.
$$

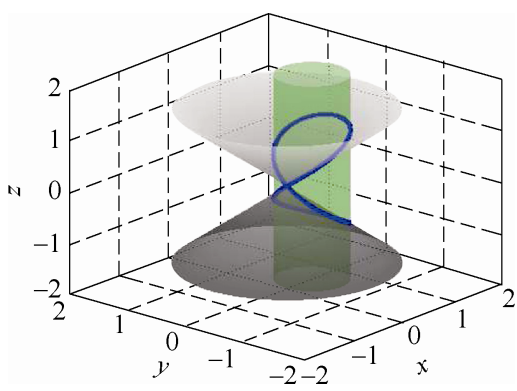

图 13 生成切线曲面的准线

以此准线生成可展切线面如图 14 所示, 其方 程为

$$
\begin{gathered}
r=\left(\cos ^{2} \theta-2 u \sin \theta \cos \theta\right), \\
\sin \theta \cos \theta-u \sin ^{2} u \cos ^{2} \theta, \\
\cos \theta-u \sin \theta)
\end{gathered}
$$

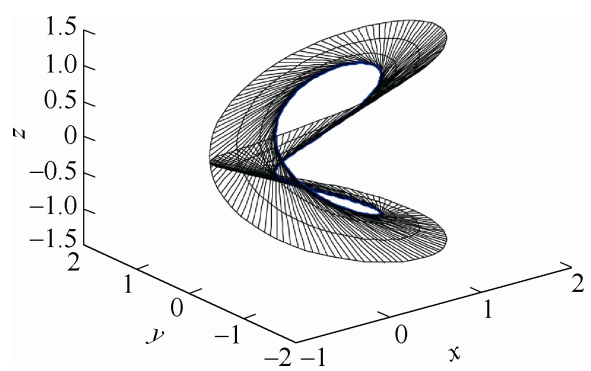

图 14 由准线生成切线面

将此切线面的法矢映射到高斯球上, 形成球面 高斯曲线如图 15 所示。

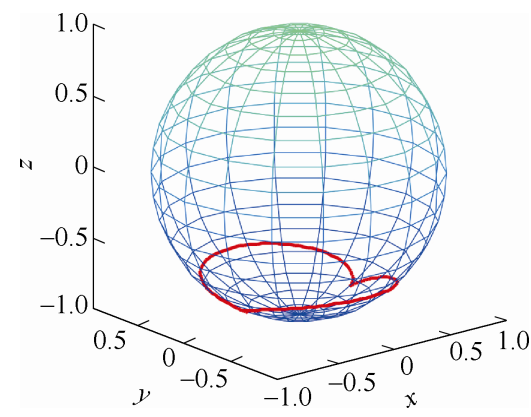

图 15 曲面映射成为高斯球面曲线

通过对高斯曲线的离散, 得到高斯曲线上离散 的曲率点, 如图 16 所示。

将高斯曲线上的离散点映射到切线面, 得到离 散后切线面上对应的直母线, 如图 17 所示。

通过算例可以看到, 通过高斯球面曲线的映 射及其生成高斯球面曲线离散, 可实现曲面直母 线离散。

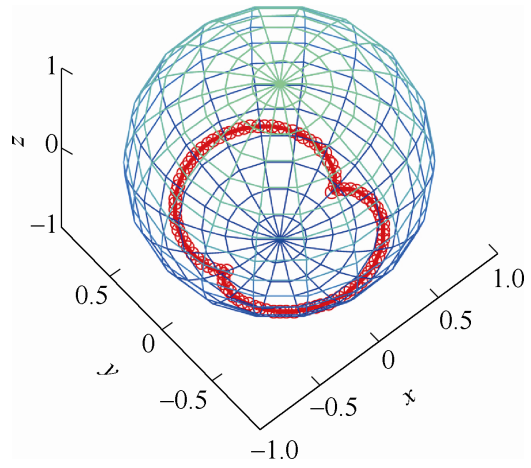

图 16 高斯球面曲线的离散

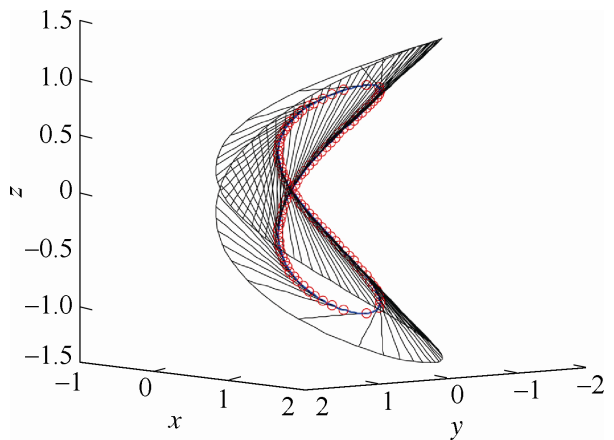

图 17 球面曲线的离散点映射回切线面的直母线并 对应实现切线曲面的等曲率离散

\section{7 结论}

（1）本文通过利用微分几何中高斯映射的概 念, 将可展曲面的特有几何性质与高斯曲线的球面 曲线性质相结合，利用将可展曲面映射为球面高斯 曲线, 实现研究曲面模型的降维处理, 并继而利用 高斯球面曲线的近似等弧长离散, 实现可展曲面的 等曲率法矢离散, 最终获得离散曲线的直母线, 为 下一步可展曲面的多面体近似建立理论基础。

(2) 单个曲面的离散问题, 通过分析高斯球面 曲线的几何意义和性质, 可以扩展到折展单元的 离散分析, 进而将多个折展曲面离散性质结合起 来。因此，高斯球面曲线性质分析对于整个折展 结构的分析建立重要的等曲率离散操作的理论依 据和条件。

\section{参 考 文 献}

[1] DAI J S, JONES J R. Kinematics and mobility analysis of carton folds in packing manipulation based on the mechanism equivalent[J]. J. Mech. Eng. Sci., 2002, 216(10): 959-970.

[2] BALKCOM D J, MASON M T. Robotic origami folding[J]. Int. J. Rob. Res., 2008, 27(5): 613-627.

[3] Online Crease Pattern Gallery, LANG ORIGAMI [EB/OL]. [2018-10-12]. https: //langorigami.com/article/ 
crease- patterns-for-folders/.

[4] CHEN Y, PENG R, YOU Z. Origami of thick panels[J]. Science, 2015, 349(6246): 396-400.

[5] HUFFMAN D A. Curvature and creases: A primer on paper[J]. IEEE Trans. Comput., 1976, C-25(10): 10101019.

[6] FUCHS D, TABACHNIKOV S. More on paperfolding[J]. Am. Math. Mon., 1999, 106(1): 27-35.

[7] REDONT P. Representation and deformation of developable surfaces[J]. Comput. Aided Des., 1989, 21(1): 13-20

[8] BO P, WANF W. Geodesic-controlled developable surfaces for modeling paper bending[J]. Comp. Graph. Forum, 2007, 26(3): 365-374.

[9] KOSCHITZ D. Computational design with curved creases: David Huffman's approach to paperfolding[D]. Boston: MIT, 2014.

[10] KILIAN M, FLOERY S, MITRA N J, et al. Curved folding[J]. ACM Trans. Graph., 2008, 27(3): 1-9.

[11] KERGOSIEN Y, GOTODA H, KUNII T. Bending and creasing virtual paper[J]. IEEE Comput. Graph. Appl.,
1994, 14(1): 40-48.

[12] WANG F, GONG H, CHEN X, et al. Folding to curved surfaces: A generalized design method and mechanics of origami-based cylindrical structures[J]. Sci. Rep., 2016, 6(1): 33312-9.

[13] TACHI T. Composite rigid-foldable curved origami structure $[\mathrm{C}] / /$ The First Conference Transformables, Sept. 18-20, 2013, Seville, Spain, 2013: 1-6.

[14] DUDTE L H, VOUGA E, TACHI T, et al. Programming curvature using origami tessellations $[\mathrm{J}]$. Nat. Mater., 2016, 15(5): 583-588.

[15] NELSON T G, LANG R J, PEHRSON N A, et al. Facilitating deployable mechanisms and structures via developable lamina emergent arrays[J]. ASME J. Mech. Rob., 2016, 8(3): 031006-9.

作者简介: 张利萍, 女, 1974 年出生, 博士, 副教授。主要研究方向为 机构学与机器人学、机械设计及其理论。

E-mail: zhangliping@westlake.edu.cn

彭彦平, 男, 1962 年出生, 博士, 教授。主要研究方向为工程设计、新 型机械设备开发。

E-mail: pengyp@dlpu.edu.cn 